\title{
A Jensen-Shannon Kernel for Hypergraphs
}

\author{
Lu Bai ${ }^{1}$, Edwin R. Hancock ${ }^{1, \star}$, and Peng Ren ${ }^{2, \star \star}$ \\ 1 Department of Computer Science \\ University of York, UK \\ Deramore Lane, Heslington, York, YO10 5GH, UK \\ ${ }^{2}$ College of Information and Control Engineering \\ China University of Petroleum (Huadong), China
}

\begin{abstract}
In this paper we explore how to construct a Jensen-Shannon kernel for hypergraphs. We commence by calculating probability distribution over the steady state random walk on a hypergraph. The Shannon entropies required to construct the Jensen-Shannon divergence for pairs of hypergraphs are obtained from steady state probability distributions of the random walk. The Jensen-Shannon divergence between a pair of hypergraphs is the difference between the Shannon entropies of the separate hypergraphs and a composite structure. Our proposed kernel is not restricted to hypergraphs. Experiments on (hyper)graph datasets extracted from bioinformatics and computer vision datasets demonstrate the effectiveness and efficiency of the Jensen-Shannon hypergraph kernel for classification and clustering.
\end{abstract}

\section{Introduction}

Hypergraph based strategies have recently been investigated for representing and processing structures where the relations present between objects are higher order. A hypergraph is a generalization of a graph. Unlike the pairwise edges in a graph, hypergraph representations allow a hyperedge to encompass an arbitrary number of vertices, and can hence capture multiple relationships among features. There have been several successful methods for characterizing hypergraphs, which include a) marginalizing higher order relationships to unary order [14], b) marginalizing the higher order relationships to pairwise order and then adopt pairwise graph matching methods [3], c) performed visual clustering by adopting tensors for representing uniform hypergraphs [10], and d) exploiting a set of coefficients from hypergraph Ihara zeta function to capture frequency of the cycle structures in a hypergraph [9]. One main limitation of the existing methods for hypergraph characterization is that they are usually limited to uniform structures, and do not fully capture hypergraph characteristics. On the other hand, existing hypergraph characterization methods also tend to require prohibitive computational overheads. In order to overcome these problems, an

\footnotetext{
${ }^{\star}$ Edwin R. Hancock is supported by a Royal Society Wolfson Research Merit Award.

** Peng Ren is supported by the National Natural Science Foundation of China Grant 61105005 .
} 
attractive alternative is to use kernel methods. Kernel methods are popular in statistical learning theory and offer an elegant way to formulate efficient algorithms to deal with high dimensional data, without the need to construct an explicit high dimensional feature space. As one of the special case of ILP [13, a number of graph kernels have been developed and proven to be powerful in graph clustering and classification. These graph kernels can be generally categorized into three classes [11, i.e. graph kernels based on a) walks, b) paths and c) restricted subgraph and subtree structures. To generalize the graph kernels to construct hypergraph kernels, Wachman has summarized the existing graph kernels based on walks and then proposed a rooted kernel for hypergraphs [13]. However, the definitions of these kernels, no matter for graphs or hypergraphs, highly rely on the enumerations of topology features such that most of them cannot be efficiently computed in an algebraic manner.

Recently, information theory has been used to define a new family of kernels based on probability distributions over the elements of the objects being compared, and these have been applied to structured data [7. These so-called nonextensive information theoretic kernels are derived from the mutual information between probability distributions on different structures, and are related to the Shannon entropy. An example is the Jensen-Shannon kernel [7. Our aim in this paper is to explore whether the Jensen-Shannon kernel can be applied to hypergraphs. The kernel is computed using the Jensen-Shannon divergence between pairs of hpyergraphs. The Jensen-Shannon divergence between a pair of hypergraphs is defined as the difference in entropies between a composite hypergraph formed from the two hypergraphs, and the sum of the entropies for the two separate hypergraphs. The required entropies are computed using the Shannon entropy with the probability distributions associated with the steady state random walks on the separate hypergraphs and their composite hypergraph. Since the probability distribution of a hypergraph can be calculated directly from the incidence matrix of the hypergraph, and the adjacency matrix of a graph can be easily converted into an incidence matrix representation. Hence, our kernel can be applied to undirected graphs. We perform experiments on several bioinformatics and computer vision datasets. We empirically demonstrate that our kernel can not only readily accommodate nonuniform hypergraphs but also easily scale to large hypergraphs. The performance of our kernel is competitive to state of the art graph kernels and hypergraph based methods.

\section{Definitions and Notations}

\subsection{Hypergraph Fundamentals}

A hypergraph is a generalization of a undirected graph, it is usually denoted by a pair set $G(V, E)$ where $V$ is a set of vertices and $E$ is a set of non-empty subsets of $V$ called hyperedges. A hypergraph can be represented in terms of a matrix. For a hypergraph $G(V, E)$ with $I$ vertices and $J$ hyperedges, its incidence matrix $\mathcal{H}$ is defined as a $I \times J$ matrix with element $\mathcal{H}(i, j)$ as follows: 


$$
\mathcal{H}(i, j)=\left\{\begin{array}{l}
1 \text { if } v_{i} \in e_{j} \\
0 \text { otherwise }
\end{array}\right.
$$

An example hypergraph is shown in Fig!1(a). Here the vertex set is $V=$ $\left\{v_{1}, v_{2}, v_{3}, v_{4}, v_{5}, v_{6}\right\}$ and the hyperedge set is $E=\left\{e_{1}=\left\{v_{1}, v_{2}, v_{3}\right\}, e_{2}=\right.$ $\left.\left\{v_{3}, v_{4}, v_{5}\right\}, e_{3}=\left\{v_{5}, v_{6}\right\}\right\}$. The incidence matrix is shown in Fig (b)

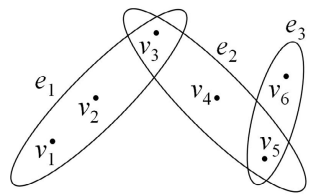

(a)

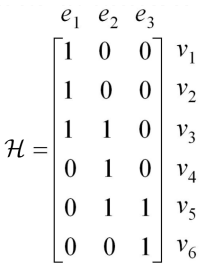

(b)

Fig. 1. (a) Hypergraph example. (b) Incidence matrix

\subsection{Jensen-Shannon Kernel}

The Jensen-Shannon kernel is a nonextensive mutual information kernel [7]. It is defined on probability distributions over structured data. The kernel for structures $p$ and $q$ is positive definite (pd) with the following kernel function

$$
k_{J S K}\left(P_{p}, P_{q}\right)=\log 2-J S D\left(P_{p}, P_{q}\right)
$$

where $J S D\left(P_{p}, P_{q}\right)$ is the Jensen-Shannon divergence between the probability distributions $P_{p}$ and $P_{q}$ defined as

$$
J S D\left(P_{p}, P_{q}\right)=H_{S}\left(\frac{P_{p}+P_{q}}{2}\right)-\frac{1}{2}\left(H_{S}\left(P_{p}\right)+H_{S}\left(P_{q}\right)\right)
$$

where $H_{S}$ is the Shannon entropy. We are interested in computing a hypergraph kernel between pairs of hypergraphs using the Jensen-Shannon divergence. For a pair of hypergraphs $G_{p}\left(V_{p}, E_{p}\right)$ and $G_{q}\left(V_{q}, E_{q}\right)$, the Jensen-Shannon divergence is given by

$$
J S D\left(P\left\{G_{p}\right\}, P\left\{G_{q}\right\}\right)=H_{S}\left(P\left\{G_{p} \oplus G_{q}\right\}\right)-\frac{H_{S}\left(P\left\{G_{p}\right\}\right)+H_{S}\left(P\left\{G_{q}\right\}\right)}{2}
$$

where $P\left\{G_{p}\right\}$ and $P\left\{G_{q}\right\}$ are the probability distributions on $G_{p}\left(V_{p}, E_{p}\right)$ and $G_{q}\left(V_{q}, E_{q}\right)$, and $P\left\{G_{p} \oplus G_{q}\right\}$ is the probability distribution on the composite hypergraph $G_{p} \oplus G_{q}$ of $G_{p}\left(V_{p}, E_{p}\right)$ and $G_{q}\left(V_{q}, E_{q}\right)$. Here $H_{S}\left(P\left\{G_{p}\right\}\right)$ is the Shannon entropy for the probability distribution $P\left\{G_{p}\right\}$ given by

$$
H_{S}\left(P\left\{G_{p}\right\}\right)=-\sum_{k=1}^{\left|V_{p}\right|} P\left\{G_{p}\right\}(k) \log P\left\{G_{p}\right\}(k)
$$

where $P\left\{G_{p}\right\}(k)$ is the $k$-th element of the probability distribution $P\left\{G_{p}\right\}$. We will use the disjoint union to construct the composite hypergraph $G_{p} \oplus G_{q}$. 


\section{Jensen-Shannon Hypergraph Kernel}

In this section, we show how to establish a Jensen-Shannon kernel for hypergraphs. We commence by defining the probability distribution associated with the steady state random walk on a hypergraph. Then we show how the probability distributions of hypergraphs can be used to compute the required Shannon entropies for the Jensen-Shannon kernel between pairs of hypergraphs.

\subsection{Probability Distribution over Steady State Random Walk}

We use the steady state random walk on a hypergraph to calculate the probability distribution for the Shannon entropy. For a hypergraph $G(V, E)$ with the incidence matrix $\mathcal{H}$ defined in (1), the vertex degree $d\left(v_{i}\right)$ for $v_{i} \in V$ is $d\left(v_{i}\right)=\sum_{e_{j} \in E} \mathcal{H}(i, j)$. Based on the definition in [4], the probability of a steady state random walk through hyperedges on $G(V, E)$ visiting vertex $v_{i}$ is

$$
P\{G\}(i)=P_{G}\left(v_{i}\right)=d\left(v_{i}\right) / \sum_{u \in V} d(u)
$$

\subsection{Composite Structure: Disjoint Union Hypergraph}

We use the disjoint union of a pair of hypergraphs as the composite structure to compute the Jensen-Shannon kernel of hypergraphs. Based on the definition in [1, the disjoint union of a pair of hypergraphs is a binary operation that combines all distinct elements of the pair of hypergraphs, while retaining the original set of memberships as a distinguishing characteristic. For a pair of hypergraphs $G_{p}\left(V_{p}, E_{p}\right)$ and $G_{q}\left(V_{q}, E_{q}\right)$, the disjoint union hypergraph $G_{U}\left(V_{U}, E_{U}\right)$ of $G_{p}\left(V_{p}, E_{p}\right)$ and $G_{q}\left(V_{q}, E_{q}\right)$ is denoted as

$$
G_{U}\left(V_{U}, E_{U}\right)=G_{p}\left(V_{p}, E_{p}\right) \cup G_{q}\left(V_{q}, E_{q}\right)=\left\{V_{p} \cup V_{q}, E_{p} \cup E_{q}\right\}
$$

where $G_{p}\left(V_{p}, E_{p}\right)$ and $G_{q}\left(V_{q}, E_{q}\right)$ is the connected components of the disjoint union hypergraph $G_{U}\left(V_{U}, E_{U}\right)$. The probabilities of the steady state random walks visiting vertices $v_{p}$ and $v_{q}$ through hyperedges in the individual components $G_{p}\left(G_{p}, G_{p}\right)$ and $G_{q}\left(G_{q}, G_{q}\right)$ of the union are $P\left\{G_{p}\right\}\left(i_{p}\right)=$ $d\left(v_{p_{i_{p}}}\right) / \sum_{u_{p} \in V_{p}} d\left(u_{p}\right)$ and $P\left\{G_{q}\right\}\left(i_{q}\right)=d\left(v_{q_{i_{q}}}\right) / \sum_{u_{q} \in V_{q}} d\left(u_{q}\right)$ respectively. A steady state random walk which departs from a vertex in one of the components is unable to visit any vertices in the other component. In the disjoint union, the probabilities of a steady state random walk departing from $G_{p}\left(V_{p}, E_{p}\right)$ and $G_{q}\left(V_{q}, E_{q}\right)$ are $\alpha_{p}=\left|V_{p}\right| /\left(\left|V_{p}\right|+\left|V_{q}\right|\right)$ and $\alpha_{q}=\left|V_{q}\right| /\left(\left|V_{p}\right|+\left|V_{q}\right|\right)$ respectively. Then the probabilities of such a steady state random walk departing from a random vertex in $G_{U}\left(V_{U}, E_{U}\right)$ and visiting vertices $v_{p_{i_{p}}}$ and $v_{q_{i_{q}}}$ in components $G_{p}\left(V_{p}, E_{p}\right)$ and $G_{q}\left(V_{q}, E_{q}\right)$ are $\alpha_{p} P\left\{G_{p}\right\}\left(i_{p}\right)$ and $\alpha_{q} P\left\{G_{q}\right\}\left(i_{q}\right)$ respectively. In this context, we obtain the probability distribution of a steady state random walk visiting vertices through hyperedges in $G_{U}\left(V_{U}, E_{U}\right)$ as

$$
P\left\{G_{U}\right\}=P_{G_{U}}=\alpha_{p} P\left\{G_{p}\right\}+\alpha_{q} P\left\{G_{q}\right\}
$$


where $P\left\{G_{p}\right\}$ and $P\left\{G_{q}\right\}$ are the probability distributions of individual components of $G_{p}\left(V_{p}, E_{p}\right)$ and $G_{q}\left(V_{q}, E_{q}\right)$ associated with their own steady state random walks respectively. The Shannon entropy of the disjoint union hypergraph $G_{U}\left(V_{U}, E_{U}\right)$ is then defined as

$$
H_{S}\left(G_{U}\right)=H_{S}\left(P\left\{G_{U}\right\}\right)=H_{S}\left(\alpha_{p} P\left\{G_{p}\right\}+\alpha_{q} P\left\{G_{q}\right\}\right)
$$

\subsection{Jensen-Shannon Kernel on Hypergraphs}

We define a Jensen-Shannon kernel on hypergraphs. Suppose the hypergraphs under consideration are represented by the set $\left\{G_{1}, \cdots, G_{p}, \cdots, G_{q}, \cdots, G_{N}\right\}$. For a pair of hypergraphs $G_{p}\left(V_{p}, E_{p}\right)$ and $G_{q}\left(V_{q}, E_{q}\right)$, we construct the disjoint union hypergraph $G_{U}\left(V_{U}, E_{U}\right)$ as the composite structure of $G_{p}\left(V_{p}, E_{p}\right)$ and $G_{q}\left(V_{q}, E_{q}\right)$. Associated with the function defined in (41) and (9), the JensenShannon hypergraph kernel based on $G_{U}\left(V_{U}, E_{U}\right)$ is defined as

$$
\begin{aligned}
& k_{J S H K}\left(P\left\{G_{p}\right\}, P\left\{G_{q}\right\}\right)=\log 2-\left(\alpha_{p}-\frac{1}{2}\right) H_{S}\left(P\left\{G_{p}\right\}\right)-\left(\alpha_{q}-\frac{1}{2}\right) H_{S}\left(P\left\{G_{q}\right\}\right) \\
& =\log 2-\frac{2\left|V_{p}\right|-\left(\left|V_{p}\right|+\left|V_{q}\right|\right)}{2\left(\left|V_{p}\right|+\left|V_{q}\right|\right)} H_{S}\left(P\left\{G_{p}\right\}\right)-\frac{2\left|V_{q}\right|-\left(\left|V_{p}\right|+\left|V_{q}\right|\right)}{2\left(\left|V_{p}\right|+\left|V_{q}\right|\right)} H_{S}\left(P\left\{G_{q}\right\}\right) \\
& =\log 2-\frac{\left|V_{p}\right|-\left|V_{q}\right|}{2\left(\left|V_{p}\right|+\left|V_{q}\right|\right)} H_{S}\left(P\left\{G_{p}\right\}\right)-\frac{\left|V_{q}\right|-\left|V_{p}\right|}{2\left(\left|V_{p}\right|+\left|V_{q}\right|\right)} H_{S}\left(P\left\{G_{q}\right\}\right)
\end{aligned}
$$

Since the probability distributions associated with the steady state random walks and the disjoint union hypergraphs of pairs of hypergraphs can be established through the incidence matrices directly, our proposed hypergraph kernel can hence accommodate both uniform and nonuniform hypergraphs.

\subsection{Algorithmic Complexity}

The computational complexity of the proposed Jensen-Shannon hypergraph kernel depends on three factors, these include 1) the construction of the disjoint union of hypergraphs, 2) the computation of probability distributions for pairs of hypergraphs and their disjoint union hupergraphs, and 3) the construction of the kernel matrices. Consider a hypergraph dataset with size $N$ and two sample hypergraphs $G_{p}\left(V_{p}, E_{p}\right)$ and $G_{q}\left(V_{q}, E_{q}\right)$ with number of vertices $m$ and $n$ respectively. The construction of the disjoint union hypergraph $G_{U}\left(V_{U}, E_{U}\right)$ of $G_{p}\left(V_{p}, E_{p}\right)$ and $G_{q}\left(V_{q}, E_{q}\right)$ requires $O\left((m+n)^{2}\right)$ operations. Then the computation of the probability distribution for the disjoint union hypergraph $G_{U}\left(V_{U}, E_{U}\right)$ requires $O(m+n)$ operations. The computations of the probability distributions from $G_{p}\left(V_{p}, E_{p}\right)$ and $G_{q}\left(V_{q}, E_{q}\right)$ require $O(m)$ and $O(n)$ operations respectively. The construction of the kernel matrix requires $O\left(N^{2} / 2\right)$ operations.

\section{Experimental Results}

\subsection{Stability Evaluation}

We commence by evaluating the stability of our Jensen-Shannon hypergraph kernel based on the disjoint unions on pairs of hypergraphs. The evaluation employs 

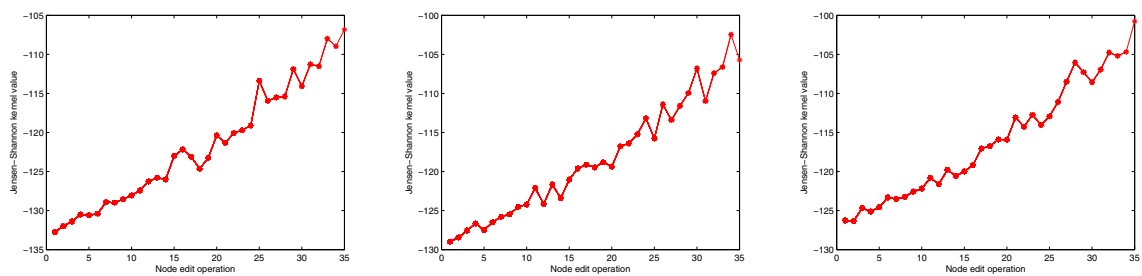

Fig. 2. Stability evaluation under hyperedge edit operation

three seed hypergraphs which have 350 vertices, 300 vertices and 250 vertices respectively. For each seed hypergraph, we perform random edit operations to simulate the effects of noise. The edit operations are based on hyperedge deletions. Since our proposed kernel can accommodate both uniform and nonuniform hypergraphs, these edit operated hypergraphs are easily accommodated by our proposed kernel. Fig, 2 shows the effects of hyperedge deletions. In each plot the $\mathrm{x}$-axis represents the fraction of hyperedges deleted, and the y-axis represents the value of the kernel $k_{J S H K}\left(G_{o}, G_{e}\right)$ between the original hypergraph $G_{o}$ and its noise corrupted counterpart $G_{e}$. The plots show that there is an approximately linear relationship between the Jensen-Shannon hypergraph kernel and the number of the deleted hyperedges, i.e. the hypergraph edit distance. This implies that our method possess the ability to distinguish hypergraphs under controlled structural-errors.

\subsection{Real-World Datasets}

We compare our proposed Jensen-Shannon hypergraph kernel (JSHK) with several alternative state of the art structural characterization methods, these include 1) hypergraph characterizations using the Ihara zeta function (HCIZF) 9], 2) the truncated Laplacian spectra (TLS) and truncated normalized Laplacian spectra (TNLS) [8], 3) the Weisfeiler-Lehman subtree kernel [12, 4) the Ramon \& Gaertner graph kernel [5], 5) the p-random walk graph kernel [6] 6 ) the random walk graph kernel [5], 7) the shortest path graph kernel [2], and 8) graphlet count graph kernel. We use three stantard graph based datasets extracted from bioinformatics datasets 12 and a hypergraph based dataset extracted from the COIL image dataset for experimental evaluation.

MUTAG: The MUTAG benchmark is based on graphs representing 188 chemical compounds, and aims to predict whether each compound possesses mutagenicity. The maximum and average number of vertices are 28 and 17.93 respectively. As the vertices and edges of each compound are labeled with a real number, we transform these graphs into unweighted graphs.

ENZYMES: The ENZYMES dataset is a dataset based on graphs representing protein tertiary structures consisting of 600 enzymes from the BRENDA enzyme database. In this case the task is to correctly assign each enzyme to one of the 6 
EC top-level classes. The maximum and average number of vertices are 126 and 32.63 respectively.

D\&D: The D\&D dataset contains 1178 protein structures. Each protein is represented by a graph, in which the nodes are amino acids and two nodes are connected by an edge if they are less than 6 Angstroms apart. The prediction task is to classify the protein structures into enzymes and non-enzymes. The maximum and average number of vertices are 5748 and 284.32 respectively.

COIL: The COIL dataset consists of images of 100 objects. In our experiments, we use selected images for three similar cups, three similar bottles and three pieces of similar vegetable. For each object we employ 18 images captured from different viewpoints. The hypergraph are extracted using feature hypergraph method [9] The maximum and minimum vertices of COIL dataset are 549 and 213 respectively.

\subsection{Experiments on Graphs Extracted from Bioinformatics Datasets}

We evaluate the performance of our kernel (JSHK) on the graphs extracted from the bioinformatics datasets. We then perform 10-fold cross-validation associated with SMO-Support Vector Machine Classification to evaluate the performance of our kernel and the alternative methods, using nine samples for training and one for testing. All parameters of the SVMs were optimized. We report the average prediction accuracies of each method in Table 1, the runtime were measured under Matlab R2011a running on a ThinkPad T61P with 2.2GHz Intel 2-Core processor with 2GB RAM. We also compare our kernel with several state of the art graph kernels. Shervashidze et al. 12 have reported the accuracies of the graph kernels based on the same classification method and datasets as ours. The runtime of these methods were measured under Matlab R2008a running on an ApplePro with 3.0GHz Intel 8-Core processor with 16GB RAM. We report these accuracies and runtimes in Table.1.

The graphs in the D\&D dataset are on average more than 284 nodes and at maximum 5748 nodes. The accuracy and runtime of our JSHK is competitive to that of the Weisfeiler-Lehman subtree kernel, graphlet count graph kernel and the shortest path kernel. The other alternative kernels did not finish in two days.

The graphs of the MUTAG dataset are of similar sizes, but correspond to very different structures. On this dataset, the accuracy of our JSHK outperforms all the other alternatives. The runtime of JSHK is competitive to that of the Weisfeiler-Lehman subtree kernel,graphlet count graph kernel and the shortest path kernel, and outperforms the other alternatives.

The graphs in the ENZYMES are of variable sizes. On this dataset, the accuracy of the JSHK is $27.05 \%$. It is lower than the accuracies of the WeisfeilerLehman subtree kernel, graphlet count kernel and the shortest path kernel, but higher than that of the other alternatives. The runtime of JSHK outperforms the alternatives.

On the whole, the accuracy and runtime of our JSHK outperforms or is competitive to that of all the alternative kernels. Only the Weisfeiler-Lehman subtree kernel and the shortest path kernel are competitive to our JSHK. 
Table 1. Performance and CPU Runtime Comparison on Bioninformatics Datasets

\begin{tabular}{c|ccc}
\hline Datasets & MUTAG & ENZYMES & D\&D \\
\hline \hline JSHK & $87.76 \%$ & $27.05 \%$ & $78.00 \%$ \\
\hline Weisfeiler-Lehman & $82.05 \%$ & $46.42 \%$ & $79.78 \%$ \\
\hline Ramon\& Gaertner & $83.78 \%$ & 13.35 & 57.27 \\
\hline p-random walk & $79.19 \%$ & $27.67 \%$ & 66.64 \\
\hline random walk & $80.72 \%$ & $21.68 \%$ & 71.70 \\
\hline shortest path & $87.28 \%$ & $41.68 \%$ & 78.45 \\
\hline graphlet count & $75.61 \%$ & $32.70 \%$ & 78.59 \\
\hline \hline Datasets & MUTAG & ENZYMES & D\&D \\
\hline \hline JSHK & $2 "$ & $19 "$ & $14^{\prime} 59^{\prime \prime}$ \\
\hline Weisfeiler-Lehman & $6 "$ & $20 "$ & $11^{\prime}$ \\
\hline Ramon\& Gaertner & $40^{\prime} 60 "$ & $38 d a y s$ & $103 d a y s$ \\
\hline p-random walk & $4^{\prime} 42^{\prime \prime}$ & $10^{\prime}$ & 4 days \\
\hline random walk & $12^{\prime \prime}$ & $12^{\prime} 19 "$ & $48 d a y s$ \\
\hline shortest path & $2 "$ & $5 "$ & $23 h 17^{\prime} 2^{\prime \prime}$ \\
\hline graphlet count & $3 "$ & $25^{\prime \prime}$ & $30^{\prime} 21^{\prime \prime}$ \\
\hline
\end{tabular}

\subsection{Hypergraph Clustering Comparisons}

In this subsection, we illustrate the clustering performance of proposed JSHK on the hypergraph dataset extracted from the COIL image dataset. We also compare our methods with several alternative state of the art hypergraph based learning methods which include HCIZF, TLS and TLNS. We compute the kernel matrices or embedding vectors using our methods and the alternatives respectively. Then we apply the $K$-means clustering method to compute the classification accuracies for the three groups of testing hypergraphs. We report the highest prediction accuracies of each method in Table.1(-:over computing, i.e.infinite value). Table.2 indicates that high accuracies for our methods are achievable. Our JSHK outperforms all the alternatives. For the cup object images based hypergraphs which the maximum and minimum vertices are 310 and 213 respectively, the accuracy of HCIZF is competitive to that of our methods. But for the bottle and vegetable object images based hypergraphs, HCIZF generates over computing (i.e.infinite value), since the maximum and minimum vertices are 549 and 305 respectively. The experiments reveal that our proposed JSHK can easily scale up even to large size hypergraph data.

Table 2. Accuracy of Classification Comparison on Hypergraphs

\begin{tabular}{c|cccc}
\hline \hline Datasets & JSHK & TLS & TNLS & HCIZF \\
\hline Cups & $97.55 \%$ & $86.60 \%$ & $52.78 \%$ & $96.29 \%$ \\
\hline Bottles & $100 \%$ & $77.41 \%$ & $83.39 \%$ & - \\
\hline Vegetable & $94.44 \%$ & $77.20 \%$ & $69.65 \%$ & - \\
\hline \hline
\end{tabular}




\section{Conclusion}

In this paper, we have shown how to construct a Jensen-Shannon kernel for hypergraphs using the Jensen-Shannon divergence. The method is based on the probability distribution over the steady state random walk on a hypergraph. We ues the Shannon entropy to measure the mutual information between a pair of hypergraphs and establish hypergraph kernel. Experimental results reveal that our kernel is competitive to the state of the art graph kernels and hypergraph based learning methods.

\section{References}

1. Berge, C.: Hypergraphs: combinatorics of finite sets. North-Holland (1989)

2. Borgwardt, K.M., Kriegel, H.P.: Shortest-path kernels on graphs. In: Proceedings of the IEEE International Conference on Data Mining, pp. 74-81 (2005)

3. Chertok, M., Keller, Y.: Efficient high order matching. IEEE Transactions on Pattern Analysis and Machine Intelligence 32, 2205-2215 (2010)

4. Cooper, C., Frieze, A.M., Radzik, T.: The Cover Times of Random Walks on Hypergraphs. In: Kosowski, A., Yamashita, M. (eds.) SIROCCO 2011. LNCS, vol. 6796, pp. 210-221. Springer, Heidelberg (2011)

5. Gärtner, T., Flach, P.A., Wrobel, S.: On graph kernels: Hardness results and efficient alternatives. In: Proceedings of the Conference on Computational Learning Theory, pp. 129-143 (2003)

6. Kashima, H., Tsuda, K., Inokuchi, A.: Marginalized kernels between labeled graphs. In: Proceedings of the International Conference on Machine Learning, pp. 321-328 (2003)

7. Martins, A.F.T., Smith, N.A., Xing, E.P., Aguiar, P.M.Q., Figueiredo, M.A.T.: Nonextensive information theoretic kernels on measures. Journal of Machine Learning Research 10, 935-975 (2009)

8. Oliva, A., Torralba, A.: Modeling the shape of the scene: A holistic representation of the spatial envelope. International Journal of Computer Vision 42, 145-175 (2001)

9. Ren, P., Aleksic, T., Wilson, R.C., Hancock, E.R.: A polynomial characterization of hypergraphs using the ihara zeta function. Pattern Recognition 44, 1941-1957 (2011)

10. Shashua, A., Levin, A.: Linear image coding for regression and classification using the tensor-rank principle. In: Proceedings of the IEEE Conference on Computer Vision and Pattern Recognition, pp. 42-49 (2001)

11. Shervashidze, N., Borgwardt, K.M.: Fast subtree kernels on graphs. In: Proceedings of the Neural Information Processing Systems, pp. 1660-1668 (2009)

12. Shervashidze, N., Schweitzer, P., van Leeuwen, E., Mehlhorn, K., Borgwardt, K.: Weisfeiler-lehman graph kernels. Journal of Machine Learning Research 1, 1-48 (2010)

13. Wachman, G., Khardon, R.: Learning from interpretations: a rooted kernel for ordered hypergraphs. In: Proceedings of the International Conference on Machine Learning, pp. 943-950 (2007)

14. Zass, R., Shashua, A.: Probabilistic graph and hypergraph matching. In: Proceedings of the IEEE Conference on Computer Vision and Pattern Recognition (2008) 